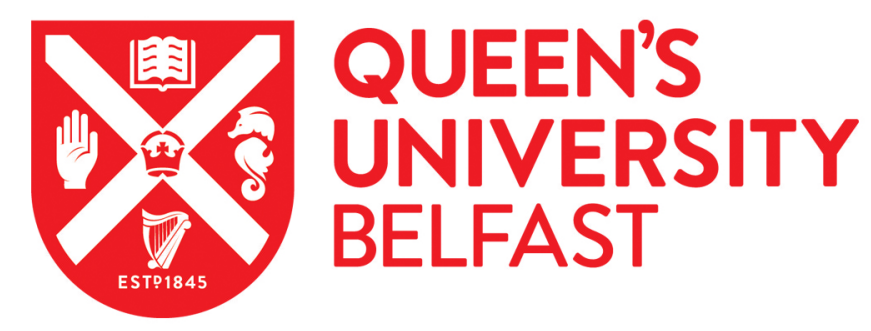

\title{
A simple measurement technique for accurate bistatic retrodirective radiation pattern calculation based on the active element pattern
} method

Buchanan, N. B., \& Fusco, V. F. (2018). A simple measurement technique for accurate bistatic retrodirective radiation pattern calculation based on the active element pattern method. IEEE Transactions on Antennas and Propagation, 66(1), 472-475. https://doi.org/10.1109/TAP.2017.2776611

Published in:

IEEE Transactions on Antennas and Propagation

Document Version:

Peer reviewed version

Queen's University Belfast - Research Portal:

Link to publication record in Queen's University Belfast Research Portal

Publisher rights

Copyright 2017 IEEE. This work is made available online in accordance with the publisher's policies. Please refer to any applicable terms of use of the publisher.

\section{General rights}

Copyright for the publications made accessible via the Queen's University Belfast Research Portal is retained by the author(s) and / or other copyright owners and it is a condition of accessing these publications that users recognise and abide by the legal requirements associated with these rights.

Take down policy

The Research Portal is Queen's institutional repository that provides access to Queen's research output. Every effort has been made to ensure that content in the Research Portal does not infringe any person's rights, or applicable UK laws. If you discover content in the

Research Portal that you believe breaches copyright or violates any law, please contact openaccess@qub.ac.uk. 


\section{A simple measurement technique for accurate bistatic retrodirective radiation pattern calculation based on the active element pattern method}

\author{
N. B. Buchanan, V.F. Fusco
}

\begin{abstract}
This paper presents a new method for calculating bistatic responses from retrodirective arrays when only monostatic measurement data is available. The proposed approach offers considerable simplification to the measurement facilities required for retrodirective array characterisation since bistatic measurements are significantly more complicated to perform in comparison to monostatic measurements. The method involves making some minor changes to the monostatic measurement configuration, enabling the capture of active element patterns with magnitude and phase data, and combining these with a new expression to obtain the bistatic calculation. Very close agreement has been obtained when compared to actual measured bistatic responses of a 10 element retrodirective patch array. In particular the predicted beam position from the new method is within $0.25^{\circ}$ at $40^{\circ}$ scan angle, compared to a disagreement of $1.5^{\circ}$ using previously reported methods.
\end{abstract}

Index Terms - Phased arrays, Microstrip antenna arrays, Adaptive arrays

\section{INTRODUCTION}

To fully understand Retrodirective Antenna Array (RDA) tracking performance there is a requirement for both bistatic and monostatic radiation patterns to be measured. The monostatic measurement provides an essential insight into the overall steering performance of the RDA over the full range of scan angles, whereas the bistatic measurement (see Fig. 1(a) for a typical setup) is needed to provide data on the individual beams formed. In terms of simplicity, and compatibility with typical far field antenna ranges, monostatic measurements (Fig. 1(b)) are relatively easy to perform since they require only a single co-located transmit and receive antenna positioned at the opposite end of the test range with respect to the RDA. The RDA can then be rotated to obtain the monostatic patterns. Whilst the monostatic patterns provide essential information of the tracking performance of the RDA, they do not provide any information of the shape of the individual beams that are being formed at a particular scan angle, meaning that, important information, such as beam pointing error and side lobe levels, is not available. To obtain this additional information, bistatic measurements must be employed. For this type of measurement the transmit antenna exciting the RDA must be held at a fixed

Manuscript received November 15, 2017. The work reported here was sponsored by UK Engineering and Physical Science Research Council grants EP/E01707X/1, EPD045835/1

Neil .B. Buchanan and Vincent Fusco are with the Institute of Electronics, Communications and Information Technology (ECIT), Queen's University Belfast, Northern Ireland Science Park, Queen's Road, Queen's Island, Belfast, United Kingdom, BT3 9DT, Tel +44 2890 971806, Fax +44 2890971702 (e-mail: n.buchanan@ecit.qub.ac.uk, v.fusco@ecit.qub.ac.uk). azimuth angle relative to the RDA during the measurement (Fig. 1(a)), and another antenna used in receive mode mounted at the opposite end of the chamber with respect to the RDA. Both the transmit and the receive antenna should be able to move relative to each other [1]. Providing this transmit antenna within the bistatic arrangement, especially within a standard antenna test range, presents many challenges. (i) The transmit antenna requires a long boom that is physically strong enough to stably hold the transmit antenna and the azimuth turntable needs to withstand the additional offset load. (ii) For large RDA's, it may not be possible to mount the transmit antenna at the required far field distance from the RDA due to restrictions in the anechoic chamber size. (iii) The transmit antenna and feed cable must have sufficiently low radar cross section to prevent interference to the main retransmit beam from the RDA. With these restrictions in mind it would appear advantageous to have a method that accurately predicts bistatic radiation patterns, whilst requiring only a monostatic measurement configuration.

In this paper we present results from a test bed of a linear 10 element RDA comprised of linear polarised patch antennas. This RDA has been previously measured for bistatic and monostatic responses [2], therefore the datasets from this antenna are used here for comparison, along with a new method of radiation pattern measurements which is compatible with monostatic configurations. The method employed is an extension to the RDA active element patterns method [3]. This paper describes new modifications to the active element patterns method, to allow it to provide accurate bistatic predictions from monostatic measurement data, thus removing the need for complicated bistatic measurements.

\section{Phase Conjugating Active Element Pattern Method}

The active element radiation pattern measurement method [4] is well known for accurate prediction of large antenna phased arrays. The method involves measuring the radiation patterns of each element individually, with the remaining elements suitably terminated. It is then possible to combine the individual element results to accurately predict the performance of the phased array under full excitation.

Active element patterns for a retrodirective array can be measured using the same practical configuration as used for monostatic measurement (Fig. 1(b)), with the exception that only one element of the array is activated for each active pattern measurement, with the other elements terminated in matched loads. In the case of an active retrodirective antenna such as the one presented here this is carried out simply by disabling the transmit function of the unused elements. 


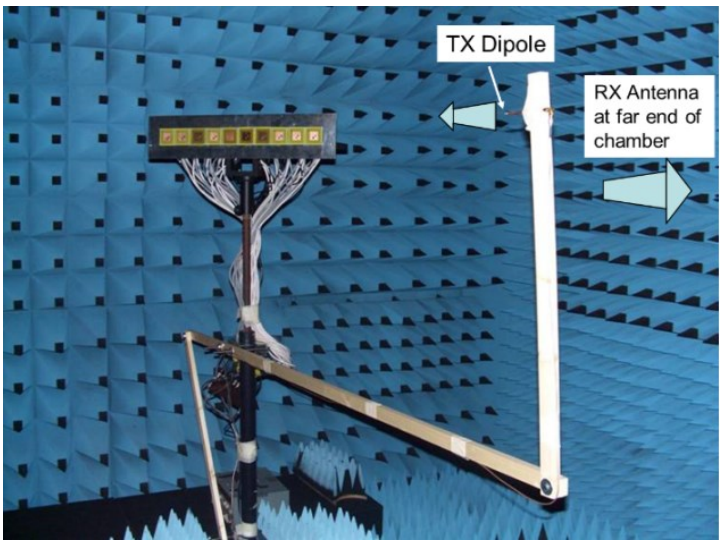

(a)

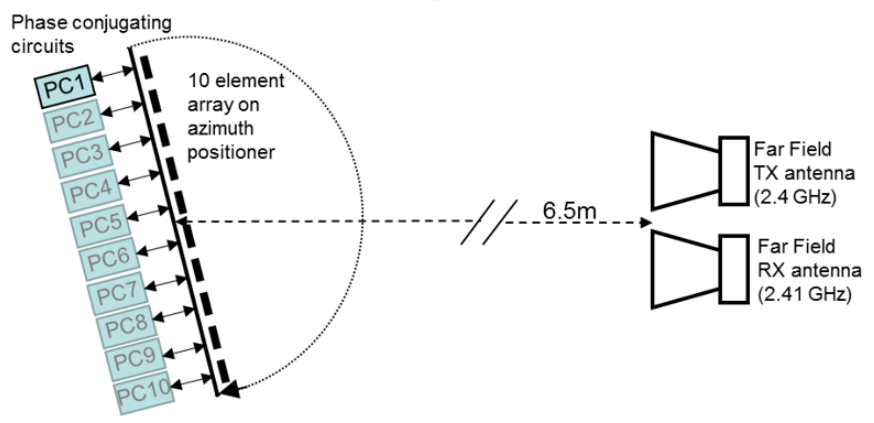

(b)

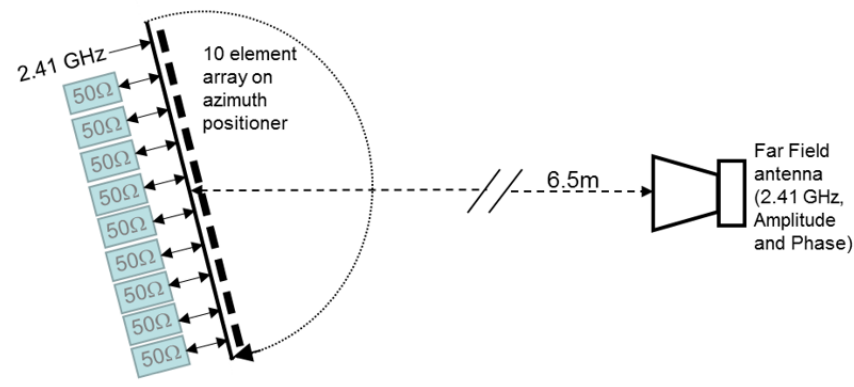

(c)

Fig. 1. Practical measurement setups for bistatic and monostatic measurements (a) Typical bistatic measurement setup

(b) Monostatic phase conjugating active element pattern (PCAEP) measurement setup showing only $\mathrm{PC} 1$ powered on

(c) New non-phase conjugating active element pattern (NCAEP) method showing element 1 measurement

We begin by noting that in an RDA the phase conjugation of the retransmit signal results in the retransmitted phase, $\phi_{t}$, of:

$$
\phi_{t(\theta)}=\phi_{r(\theta)}-\phi_{r(\theta)}+\zeta+\psi_{(\theta)}=\zeta+\psi_{(\theta)}
$$

$\zeta$ is a constant produced by the local oscillator phase of the conjugation circuit and is independent of the azimuth angle, $\theta$, $\psi_{(\theta)}$ is the conjugation error resulting from electronic circuits defects and is usually very small. Since the phase variation produced from the individual element is essentially lost in the phase conjugation process, the only useable result obtained from the phase conjugating active element patterns is magnitude. From this available data, the best prediction of the bistatic patterns can be obtained from appropriately multiplying the magnitude of the relevant active element patterns with the array factor. We call this method the phase conjugating active element pattern (PCAEP), and it can be calculated from (2), for a given bistatic scan angle, $\theta_{0}$, over a range of azimuth angles, $\theta$. The expression is derived from the response of a uniform linear array [5] multiplied by the magnitude of the RDA active element patterns, $G_{n}(\theta)$. The expression has also been modified from that of [2] to include the effect of beam pointing error caused by the frequency difference between transmit and receive wavelengths, $\lambda_{t}$ and $\lambda_{r}$.

$\sum_{n=1}^{N} G_{n}(\theta) e^{j\left[\frac{2 \pi d}{\lambda_{t}}(n-1)\left(\sin \theta-\frac{\lambda_{t}}{\lambda_{r}} \sin \theta_{0}\right)\right]}$

The results of the PCAEP prediction are shown in Fig. 2 at $0^{\circ}$ scan angle compared with the actual measured bistatic patterns. From these results, it is seen that this method has good accuracy at $0^{\circ}$ scan angle in terms of beamwidth and retransmitted beam position. Fig. 3 shows the PCAEP prediction at $-40^{\circ}$ scan angle. Here it is observed that the prediction is significantly less accurate, in terms of both the predicted steering angle and the beam shape. In particular, beam asymmetry, a well known effect observed when scanning phased arrays away from boresight [6], is not modelled by this method.

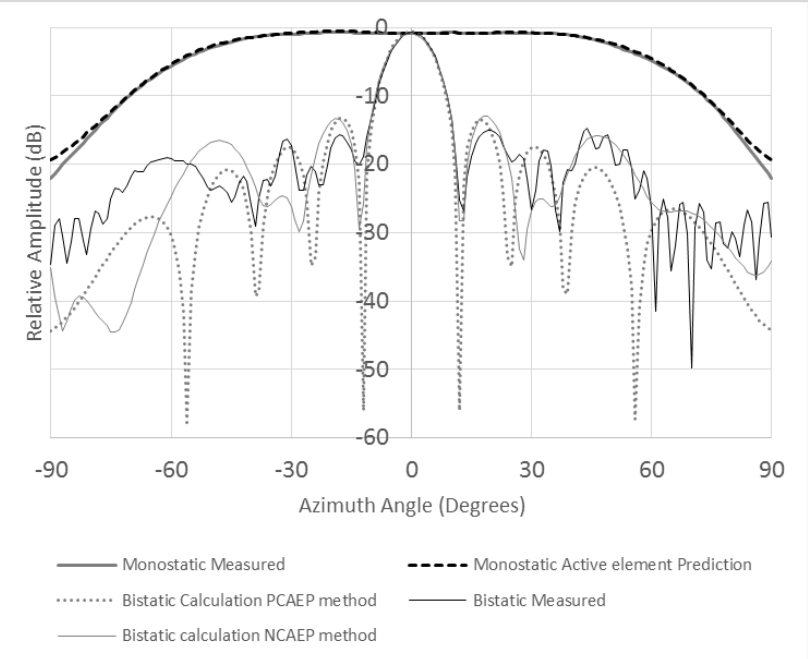

Fig. 2. Bistatic predictions at $0^{\circ}$

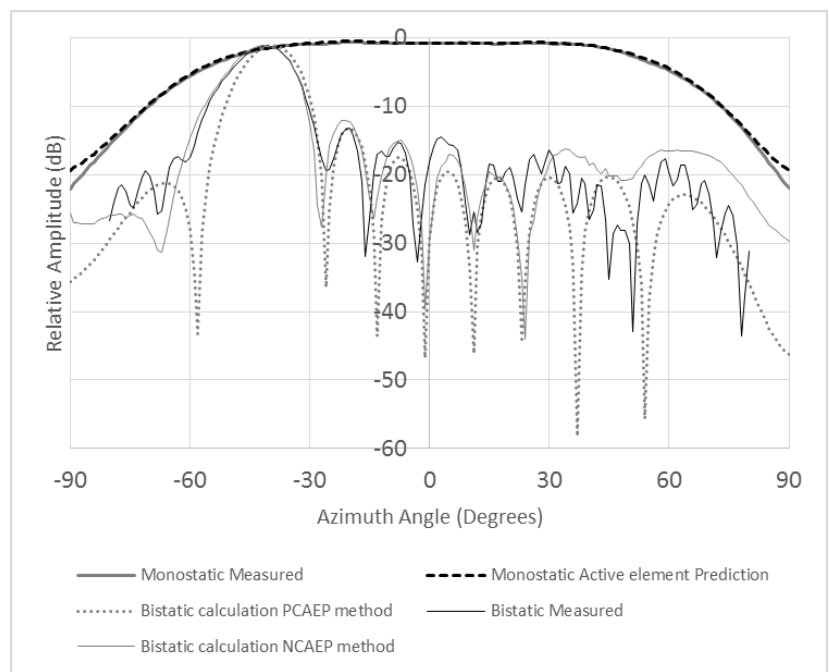

Fig. 3. Bistatic predictions at $-40^{\circ}$ 


\section{Non-Phase Conjugating Active Element Pattern METHOD}

From the previous section it is concluded that the phase conjugating active element pattern (PCAEP), cannot be used to accurately calculate bistatic responses at scan angles away from boresight. This is likely to be due to the lack of the phase information on the active element pattern responses, which is lost due to the phase conjugation process. In order to obtain the phase information the measurement configuration was modified to permit measurement of the individual elements of the array as passive patches (Fig. 1(c)), i.e. no element local phase conjugation occurs. We refer to this method as the non-phase conjugating active element pattern method (NCAEP). The measurement is still simple to accomplish, as it only requires disconnection of the phase conjugating circuit to provide direct access to each of the individual patch antenna feed ports. For large arrays, the activation of each individual element (and termination of unused elements) would benefit from some automation within the measurement setup, due to the large number of measurements that need to be obtained. This is still a viable option, particularly if the antenna test range cannot physically accommodate a bistatic measurement setup due to space constraints, or other restrictions.

The amplitude and phase responses of a single element (Element 1) measured with the NCAEP method is shown in Fig. 4. Comparing this result to the PCAEP method shows that the amplitude response of the element remains unchanged, however the phase variation with azimuth angle has now been preserved. Fig. 4 (a) also shows the radiation pattern of a centre element (Element 5) to appreciate that this gives a different response to that of the edge element (Element 1)

From the active element patterns, obtained from the NCAEP method, the bistatic radiation patterns, at a certain scan angle, $\theta_{0}$, with element spacing, $d$, can be calculated as:

$\sum_{n=1}^{N} G_{n}(\theta) e^{j\left[\alpha_{(\theta)}+\frac{2 \pi \mathrm{d}}{\lambda_{r}}(n-1) \sin \theta_{0}-\psi_{(\theta)}\right]}$

Where $\alpha_{(\theta)}$ is the measured phase information from the NCAEP method, normalised at boresight. Also included is the conjugation error, $\psi(\theta)$ from the PCAEP method, which was found to further enhance the accuracy of the prediction.

To allow (3) to model beam pointing error due to TX/RX frequency offset, the NCAEP radiation patterns were measured at the transmit frequency, $2.41 \mathrm{GHz}$, whereas the wavelength, $\lambda_{r}$ corresponding to the receive frequency, $2.4 \mathrm{GHz}$, is used in the calculation of (3). The radiation pattern results from the NCAEP method at a $-40^{\circ}$ bistatic angle is shown in Fig. 3. This shows the NCAEP method to provide an excellent prediction of the bistatic response in terms of steering angle and main beam shape, in this case beam asymmetry is in good agreement with the measured bistatic response.

One of the most notable factors that was not accurately predicted by the PCAEP method was the retransmitted beam position at azimuth angles away from boresight. To quantify this, the beam pointing error was calculated from the measured bistatic response and compared to the PCAEP and NCAEP methods in Fig. 5. The practical measurement and the NCAEP methods are in good agreement, showing the physical RDA's retransmit beam is oversteered from boresight, as caused by the effect of beam asymmetry. This effect is not modelled by the PCAEP method which predicts an understeer on the retransmitted beam, showing that the PCAEP method is not sufficient to produce accurate beam pointing calculations. Fig. 5 shows, that at $40^{\circ}$ from boresight, the main beam position differs by as much as $1.5^{\circ}$ from the PCAEP and NCAEP methods. The actual pointing loss obtained for a given beam squint depends on the beamwidth of the main beam. When predicting RDAs with large numbers of elements, i.e. high directivity as required for example in satellite applications, [7], then even a small beam squint can cause a high pointing loss. The NCAEP method is thus seen to be an essential tool when predicting accurate bistatic responses when only a monostatic measurement setup is available.

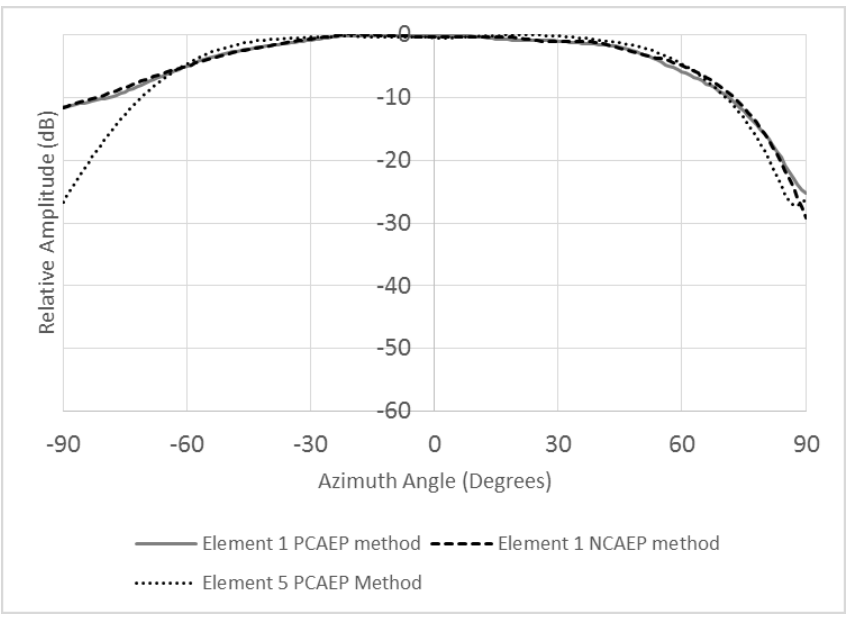

(a)

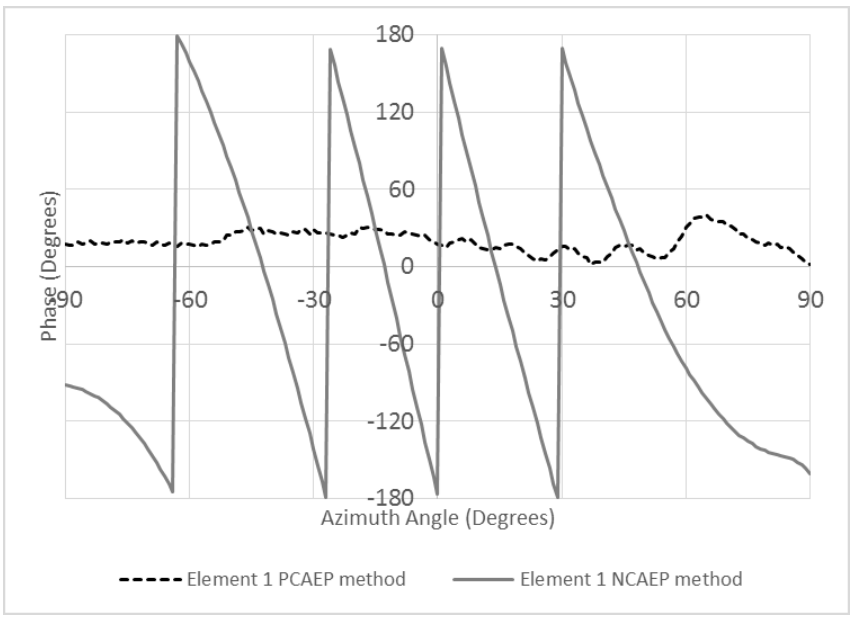

(b)

Fig. 4. Amplitude (a) and phase (b) responses of element 1 obtained using PCAEP and NCAEP methods 


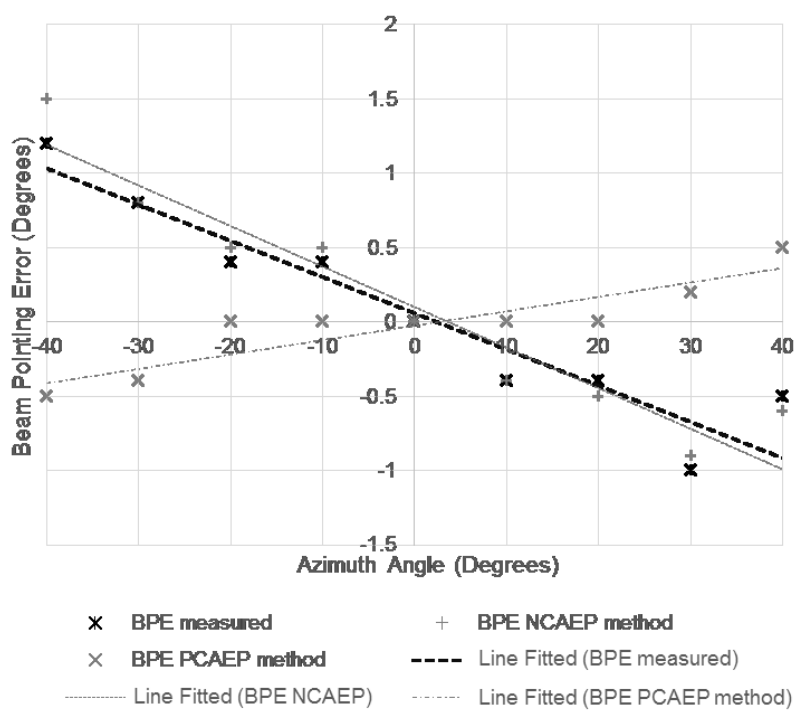

Fig. 5. Bistatic Beam pointing error (BPE) predictions

\section{CONCLUSION}

This paper has shown a new method for calculating bistatic responses of a retrodirective array, using only data that was obtained from only monostatic measurements, thus removing the need to carry out more complicated bistatic measurements. It was shown that previous methods for calculating bistatic patterns, which relied on monostatic data with no phase information, significantly degraded the predictions when steering away from boresight. Previous active element pattern predictions, for a 10 element patch array, beam pointing error predictions could differ by as much as $1.5^{\circ}$ and prediction of beam asymmetry was non-existent. The new methods shown here are accurate to $0.25^{\circ}$ beam pointing error at $-40^{\circ}$ from boresight. It is anticipated that the methods in this paper will allow accurate bistatic prediction of much larger retrodirective arrays, which would normally require complex measurement configurations to obtain the bistatic responses.

\section{ACKNOWLEDGMENT}

The authors would like to acknowledge the efforts of $\mathrm{Mr}$ Michael Major and Mr Kieran Rainey for performing the measurements in this paper.

\section{REFERENCES}

[1] C. Y. Pon, "Retrodirective Array Using the Heterodyne Technique", IEEE Trans. on Antenna and Propagation, vol. AP-12, pp. 176-180, 1964.

[2] V. Fusco, N. B. Buchanan, "High-Performance IQ Modulator-Based Phase Conjugator for Modular Retrodirective Antenna Array Implementation," IEEE Transactions on Microwave Theory and Techniques, Volume: 57 , Issue 10, Part 1, October 2009, Page(s): 2301 2306.

[3] B. Y. Toh, V. F. Fusco and N. B. Buchanan, "Retrodirective array tracking prediction using active element characterisation," in Electronics Letters, vol. 37, no. 12, pp. 727-728, 7 Jun 2001.

[4] D. M. Pozar, "The active element pattern," in IEEE Transactions on Antennas and Propagation, vol. 42, no. 8, pp. 1176-1178, Aug 1994.

[5] R.C. Hansen, "Phased Arrays Antennas," Second Edition, John Wiley \& Sons Inc, pp 7-9.
[6] W. H. Von Aulock, "Properties of Phased Arrays," in Proceedings of the IRE, vol. 48, no. 10, pp. 1715-1727, Oct. 1960.

[7] N.B. Buchanan, V.F. Fusco, M. van Der Vorst, "A High Performance Circular Polarised Retrodirective Antenna with Basic Array Function for Service Activated SATCOM Systems" European Conference on Antennas and Propagation 2012, April 2012, Prague. 